\title{
Trastornos de memoria y de atención en disfunciones cerebrales del niño
}

\author{
J. Narbona, N. Crespo-Eguílaz
}

\author{
MEMORY AND ATTENTION IN CHILDREN WITH BRAIN DYSFUNCTION
}

\begin{abstract}
Summary. Introduction. For conscious behaviours to continue, attention and working memory must be brought into play in order to match the data from the current reality with those in the long-term memory and to the sequential plans for adaptive action. Patients and methods. We present the preliminary findings of our study of 50 patients between 7 and 16 years of age, who were divided into three groups according to their dominant neurocognitive disorder: a 'pure' ADHD group, an ADHD/LD group (with comorbid learning disorder) and an RMEM (risk of memory disorder due to bilateral temporomesial damage) group. All of them were administered a battery of tests designed to evaluate attentional control and short and long-term memory. Results. Verbal IQ was above 80 in all the participants, with no differences among the three groups. The ADHD/LD group had a manipulative IQ and level of sustained attention below those of the ADHD group, but no long-term memory deficit was observed in either of these two groups. In contrast, the RMEM group's performance was poorer in verbal memory tasks, although their behaviour is more attentive and stable compared to that of the other two groups. Conclusions. Neuropsychological analysis of these dissociations allows adjustments to be made in the strategies for therapeutic interventions. Performance in long-term learning in children with $A D H D$ and $A D H D / L D$ can be improved with psychostimulants owing to the activating effect they have on working memory. [REV NEUROL 2005; 40 (Supl 1): S33-6]
\end{abstract}

Key words. ADHD. Attention. Bitemporal damage. Childhood. Memory.

\section{INTRODUCCIÓN}

La atención y la memoria de trabajo son los recursos intrumentales de la consciencia que permiten la concentración y la continuidad en el tiempo de las operaciones cognitivas y de las conductas intencionales. Más en concreto, la focalización de la consciencia sería lo que hoy conocemos como atención selectiva, desde la clásica concepción de Williams James en 1890 [1]. La continuidad, incluso ardua, de la conducta consciente dirigida a un fin, corresponde con lo que denominamos atención sostenida; ésta se apoya en la memoria de trabajo, o memoria operativa (MO), que se encarga de manipular la información actual y de relacionarla con la información de la memoria a largo plazo (MLP), de naturaleza procedimental o declarativa, relevante para la conducta en curso y para el plan de acción futura [2]. La atención no sólo juega un papel facilitador de las representaciones, sino que también, y fundamentalmente, realiza un control inhibidor de los datos irrelevantes o interferidores [3].

Si la atención se pone en juego desde los instantes iniciales de cualquier actividad perceptiva, la MO actúa en paralelo con los mecanismos atencionales para posibilitar el procesamiento controlado de la información. Tanto la atención selectiva, en sus modalidades activadora e inhibidora, como la MO, son pilares del sistema ejecutivo, en el que se implican los colículos, el sistema reticular activador, el tálamo, los ganglios de la base y las cortezas prefrontal y parietal posterior [4]. Por su parte, los procesos de adquisición, retención-consolidación y recuperación de las informaciones dependen de las estructuras mesiotemporales y demás componentes del sistema de Papez hasta el núcleo anterior del tálamo, antes de volver a proyectarse en el neocórtex. La

\footnotetext{
Aceptado: 30.01.05.

Unidad de Neurología Pediátrica. Clínica Universitaria de Navarra. Pamplona, Navarra, España.

Correspondencia: Dr. Juan Narbona. Unidad de Neurología Pediátrica. Departamento de Pediatría. Clinica Universitaria de Navarra. Avda. Pío XII, s/n. E-31080 Pamplona (Navarra). E-mail: jnarbona@unav.es

(C) 2005, REVISTA DE NEUROLOGÍA
}

memoria implícita (no declarativa) se inscribe en circuitos corticoestriotalamocorticales y corticocerebelotalamocorticales [5].

Así pues, el sistema ejecutivo y los sistemas de memoria a largo plazo se complementan a través de la memoria operativa $[6,7]$; pero uno y otro sistema se asientan en estructuras cerebrales distintas. Por tanto, se pueden observar disociaciones entre las competencias ejecutivas y las de memoria en sujetos con disfunción o daño de las estructuras prefrontales o de las mesiotemporales [8]. Las relaciones y disociaciones entre la memoria y la atención en patologías neuropsicológicas infantojuveniles sólo se han reflejado en la bibliografía en años recientes. En este trabajo aportamos los resultados preliminares de nuestra propia experiencia referentes a las capacidades de memoria declarativa y de control ejecutivo en niños con trastorno por déficit de atención e hiperactividad (TDAH) y en niños con quejas de memoria por daño cerebral, y revisamos los datos relevantes de la literatura al respecto.

Siendo el objetivo de nuestra investigación estudiar una posible disociación entre los sistemas de atención y los de memoria a largo plazo en niños y adolescentes, las hipótesis a contrastar eran: a) Los sujetos con patología mesiotemporal pueden presentar trastorno prominente de memoria pero sin problemas significativos del control atencional; y b) El TDAH sin trastornos comórbidos no conlleva merma de la memoria explícita a largo plazo.

\section{PACIENTES Y MÉTODOS}

Hemos estudiado prospectivamente la atención y la memoria en 50 sujetos con edades comprendidas entre 7 y 16 años, atendidos en la Unidad de Neurología Pediátrica de la Clínica Universitaria de Navarra. El cociente intelectual (CI) verbal en la WISC-R [9] es igual o superior a 80 en todos los sujetos. Según el principal problema referido a las capacidades neurocognitivas, los sujetos se distribuyen en tres grupos:

- Grupo con trastorno por déficit de atención e hiperactividad (TDAH, $n=$ 25, con 21 niños y 4 niñas), subtipo combinado, según los criterios de la DSM-IV [10], sin otros trastornos comórbidos, con CI verbal medio de 101 (rango: 85-120). 




Figura 1. Perfil neuropsicológico de los grupos TDAH (barras negras) y TDAH/TA (barras grises) en puntuaciones $t$ (media: 50; DE: 10). Análisis de varianza entre ambos grupos $\left({ }^{a} p<0,05\right)$.

- Grupo con TDAH más trastorno de aprendizaje de la coordinación y/o de la lectura (TDAH-TA, $n=17$; con 10 niños y 7 niñas); el cociente intelectual verbal medio es 94 (rango: 80-123).

- Grupo a riesgo de trastornó de memoria (RMEM, $n=8$; con 7 niños y 1 niña), ciertamente heterogéneo en cuanto a la base patológica, pero teniendo en común un daño relativamente selectivo de estructuras mesiotemporales; el CI verbal medio de este grupo es 96 (rango: 85-112). Se incluyen aquí 4 sujetos con antecedente de gran prematuridad y perfil de daño neuronal bitemporomesial en la espectrografía por resonancia magnética; en dos pacientes con antecedente de encefalitis herpética se constató por resonancia magnética daño bitemporal residual; en dos sujetos, afectos de epilepsia refractaria, se observó hipometabolismo bitemporal intercrítico mediante PET-fluorodesoxiglucosa.

Tras la recogida de anamnesis y la aplicación de escalas DSM-IV-RS (padres) y EDAH [11] (profesores), se realizó un perfil de inteligencia con la WISC-R y se aplicaron escalas específicas de memoria en la vida cotidiana (Rivermead Behavioural Memory Test) [12], de memoria verbal a corto y largo plazo (CAVLT-2 Children's Auditory Verbal Learning Test-2) [13] y de memoria visual a largo plazo de la Figura compleja de Rey [14]. Como medida de control de la atención y de la impulsividad se usó la modalidad prolongada a seis minutos del Test de Caras según nuestros propios baremos [15]. Asimismo, se aplicaron pruebas de conciencia fonológica, lectura, escritura y cálculo, cuyo detalle y resultados no forman parte de los objetivos de este trabajo.

Se realizó el estudio comparativo de los resultados en los tres grupos mediante ANOVA de las puntuaciones $t$ de cada grupo frente a los demás, tras prueba de normalidad, en cada una de las medidas. Se aplicó el test $\chi^{2}$ para comprobar el grado de asociación de los efectos de primacía y de recencia en el aprendizaje de listas de palabras por parte del grupo TDAH.

\section{RESULTADOS}

Mientras que el CI verbal se mostraba en torno a la media normal en ambos grupos TDAH y TDAH/TA, el CI manipulativo era significativamente inferior $(p<0,05)$ en el grupo TDAH-TA. La conducta abierta correspondiente a déficit de atención e hiperactividad-impulsividad en los medios familiar y escolar (DSM-IV-RS y EDAH-Conners) resultó más acentuada en el grupo TDAH, pero en cambio el rendimiento en la tarea estructurada de atención sostenida caras-6 fue más bajo en el grupo TDAH-TA. Estos dos grupos de sujetos, en cambio, no se mostraron diferentes en las tareas de memoria verbal y visual a corto y largo plazo (Fig. 1).

Comparando el grupo de pacientes a riesgo de trastorno de memoria RMEM con el conjunto de sujetos incluidos en ambos grupos de TDAH 'puro' y asociado a trastorno de aprendizaje (TDAH + TDAH/TA), se observa una disociación inversa de los rendimientos (Fig. 2): el grupo RMEM muestra una conducta abierta normal y significativamente superior $(p<$

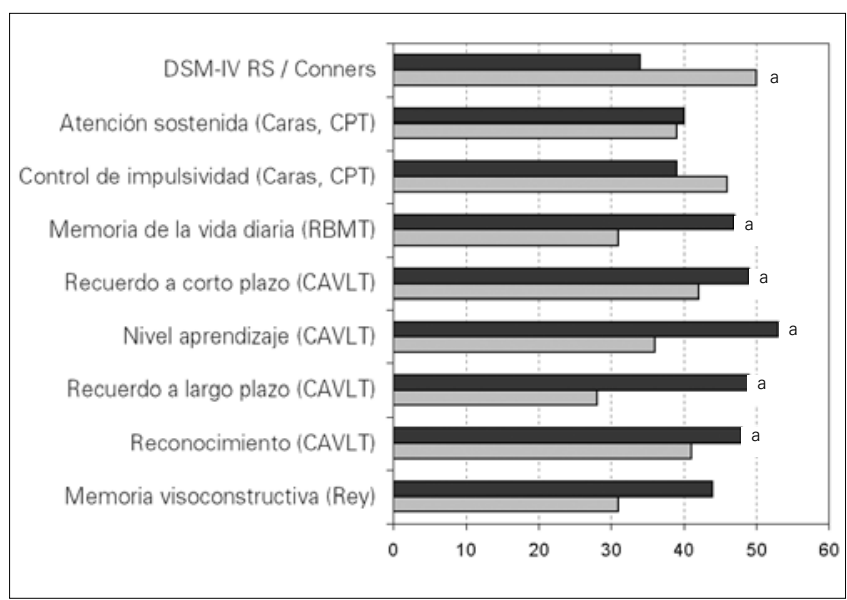

Figura 2. Perfil neuropsicológico de los grupos TDAH + TDAH/TA (barras negras) y RMEM (barras grises) en puntuaciones $t$ (media: 50; DE: 10). Análisis de varianza entre ambos grupos $\left({ }^{a} p<0,05\right)$

0,05 ) en cuanto a atención y control de impulsividad frente a los de la agrupación TDAH + TDAH/TA, pero dicho grupo RMEM, de acuerdo con lo hipotetizado, tiene un rendimiento inferior $(p<0,05)$ en tareas de memoria a corto y a largo plazo, referidas a la vida cotidiana (Rivermead) y al aprendizaje de una lista de palabras. En la reproducción de memoria de la figura compleja de Rey, la puntuación tiende a ser más alta en el grupo TDAH que en el TDAH/TA y, en ambos, superior a la del grupo RMEM, pero sin alcanzar significación estadística (Figs 1 y 2).

La estrategia utilizada por 23 sujetos del grupo con TDAH 'puro' en el recuerdo libre a corto plazo de las dos listas de palabras del CALVT no fue diferente de lo observado en población normal: así, el efecto de primacía -retención por autorrepetición de las primeras palabras de la serie- se hizo manifiesto para la primera lista, mientras que el efecto de recencia predominó para la segunda lista-devolución de las últimas palabras de la serie, usando la memoria operativa-, pues la capacidad de evocación por autorrepetición quedaba saturada por los cinco ensayos de la primera lista $\left(\chi^{2}: p<0,05\right)$.

\section{DISCUSIÓN}

La atención centrada en las representaciones internas de la memoria a largo plazo es necesaria para los procesos de pensamiento y de comunicación. La atención sostenida y la memoria operativa actúan sinérgicamente para dar continuidad y congruencia a la actividad mental y a la conducta. Si la atención y la MO cooperan de forma continua e imbricada en los procesos conscientes de la vida cotidiana, se ha podido demostrar que la atención desde los datos externos (down-top) está fuera de juego en ciertas situaciones clínicas en las que, sin embargo, el sujeto puede procesar, sin percatarse, los rasgos superficiales de objetos presentes y atribuirles un valor semántico en tareas de categorización y emparejamiento. Así ha sido demostrado en algunos pacientes con 'visión ciega' tras lesiones de la corteza primaria visual, en afectos de agnosia visual aperceptiva, de heminegligencia espacial, de prosopoagnosia o de alexia pura [16]. La agnosia espacial unilateral puede abarcar no sólo los objetos reales presentes, sino también las memorias de los objetos situados en el hemiespacio subjetivo (visual, auditivo o táctil, externo o somático) que el paciente ignora [17]. Es preciso destacar una vez más que, tanto la atención como la memoria, son capacidades muy frágiles que se pueden afectar de manera inespecífica y simultánea en múltiples patologías cerebrales.

Varios trabajos han demostrado que los sujetos con TDAH presentan déficit de rendimiento en paradigmas experimentales 
que ponen en juego la memoria operativa-visuoespacial principalmente- y que su rendimiento mejora bajo la acción de fármacos psicoestimulantes [18,19]. En cambio, la memoria a largo plazo no parece estar afectada por lo general en este tipo de pacientes, salvo en algunos casos en los que pueden evidenciarse dificultades de acceso a las representaciones, a causa de la disfunción ejecutiva [20]. En cambio, los pacientes con amnesia por disfunción bitemporal no presentan necesariamente fallo ejecutivo; por tanto, su memoria operativa y su atención pueden funcionar bien en las actividades a corto plazo.

Los resultados preliminares de nuestra investigación van a favor de una relativa independencia de los procesos de memoria a largo plazo y los de control atencional-ejecutivo. Nuestro grupo de pacientes con daño bilateral de estructuras temporomesiales mostraba un rendimiento muy bajo en memoria declarativa y aprendizajes a largo plazo, en la vida cotidiana y en los tests estructurados, de acuerdo con lo descrito en la literatura en referencia a adultos y niños [21-28], pero sin embargo no se han objetivado trastornos significativos del control atencional o de la memoria operativa en estos sujetos. Las epilepsias con abundante actividad paroxística intercrítica plantean problemas complejos de disfunción cognitiva: así, las capacidades ejecutivas y de memoria se pueden ver afectadas simultáneamente, pero en ciertos síndromes epilépticos idiopáticos la disfunción ejecutiva se puede afectar de forma prominente [29], mientras que en otras, generalmente sintomáticas, el fallo de memoria domina el cuadro neuropsicológico [30-32], como en los dos pacientes epilépticos incluidos en nuestro grupo RMEM.

En nuestro grupo de niños con TDAH 'puro', la fragilidad de la atención se manifiesta a lo largo de la conducta abierta cotidiana y en tareas arduas que requieren un especial esfuerzo del sistema ejecutivo y que no se enmarcan en las valencias motivacionales naturales del sujeto; pero, sin embargo, estos sujetos realizan con éxito tareas que le demandan menos esfuerzo sostenido de la atención y de la memoria operativa (ejemplo de la 'prótesis cognitiva' que representan los video-juegos), y también han puntuado normalmente en una tarea de atención sostenida bajo situación estructurada (caras-6). En cambio, nuestros pacientes con TDAH más trastorno de aprendizaje comórbido -la mayoría de ellos, con un perfil de trastorno de aprendizajes no verbales- han mostrado un rendimiento más bajo en la tarea de control ejecutivo que los afectos de TDAH 'puro'. En lo que respecta a la memoria verbal y visual a largo plazo, el rendimiento tiende a ser más bajo en el grupo TDAH/ TA, pero la diferencia no es significativa.

La observación, en el grupo con TDAH, de los efectos de primacía y de recencia en la evocación libre de dos listas de palabras del Children's Auditory Verbal Learning Test tiene, a nuestro juicio, un particular interés: el predominio del efecto primacía al evocar la primera lista indica que los recursos de memoria por autorrepetición son adecuados; posteriormente, al incidir la segunda lista de palabras, la capacidad para memorizarla a largo plazo por autorrepetición se encuentra saturada y los sujetos utilizan como principal recurso de evocación la memoria operativa de los últimos elementos, es decir, ahora predomina el efecto de recencia. Esto quiere decir que los sujetos con TDAH sin otros problemas comórbidos poseen estrategias de memoria normales para aprender, en situación estructurada; lo cual constituye un argumento más a favor de los beneficios que puede aportar el entrenamiento del autocontrol para estos sujetos, en paralelo con el tratamiento farmacológico.

En conclusión, el sistema ejecutivo y los sistemas de memoria van de la mano y se influyen mutuamente en situaciones normales y patológicas. Algunos trabajos recientes muestran que los niños con TDAH tienen dificultades de memoria operativa mejorables con fármacos psicoestimulantes, pero los resultados preliminares de nuestro estudio muestran que el control atencional y la memoria verbal a largo plazo pueden afectarse de manera disociada: los niños con TDAH no presentan problemas de memoria verbal a largo plazo y utilizan estrategias de memoria a corto plazo normales; los que padecen TDAH más trastorno de aprendizaje suelen tener un rendimiento inferior en tareas ejecutivas. Por otra parte, los niños con daño temporomesial bilateral, junto a déficit graves de memoria declarativa, presentan una atención y resto de funciones ejecutivas normales. Estos resultados provisionales van a favor de la doble disociación que hipotetizábamos, y pueden ser de utilidad en la selección de estrategias de intervención psicopedagógica.

\section{BIBLIOGRAFÍA}

1. James W. The principles of psychology. Cambridge MA: Harvard University Press; 1890.

2. Fuster JM. The prefrontal cortex -an update: time is of the essence. Neuron 2001; 30: 319-33.

3. Barkley RA. ADHD and the nature of self-control. New York: Guilford Press; 1997.

4. Sánchez-Carpintero R, Narbona J. Revisión conceptual del sistema ejecutivo y su estudio en el niño con trastorno por déficit de atención e hiperactividad. Rev Neurol 2001; 33: 47-53.

5. Crosson B. Subcortical functions in language and memory. New York: Guilford Press; 1992

6. Flechter PC, Henson RNA. Frontal lobes and human memory. Insights from functional neuroimaging. Brain 2001; 124: 849-81.

7. Wheeler MA, Stuss DT, Tulving E. Toward a theory of episodic memory: the frontal lobes and autonoetic consciousness. Psychol Bull 1997; 121: 331-54.

8. Owen AM, Morris RG, Sahakian BJ, Polkey CE, Robbins TW. Double dissociations of memory and executive functions in working memory tasks following frontal lobe excisions, temporal lobe excisions or amygdalo-hippocampectomy in man. Brain 1999; 119: 1597-615.

9. Wechsler D. Escala de inteligencia Wechsler para niños, revisada (WISC-R). Madrid: TEA; 1993.

10. American Psychiatric Association. Manual diagnóstico y estadístico de los trastornos mentales. 4 ed. (DSM-IV). Barcelona: Masson; 1995.
11. Farré-Riba A, Narbona J. EDAH, escalas para la evaluación del trastorno por déficit de atención con hiperactividad. Manual de uso y hojas de respuesta. 5 ed. Madrid: TEA; 2004.

12. Wilson B, Cockburn J, Baddeley A. The Rivermead behavioural memory test for children aged 5-10. Suffolk: Thames Valley; 1991.

13. Talley JL. Children's Auditory Verbal Learning Test-2. Professional manual. Odessa, FL: Psychological Assessment Resources; 1996.

14. Rey A. Test de reproducción de una figura compleja. Manual, versión española, 4 ed. Madrid: TEA; 1984.

15. Crespo-Eguílaz N, Narbona J, Peralta F, Repáraz MR. Medida de la atención sostenida y del control de la impulsividad en niños: nueva modalidad de aplicación del Test de Caras. Infancia y Aprendizaje 2005 [in press].

16. Farah MJ. Consciousness. In Rapp B, ed. The handbook of cognitive neuropsychology. Hove, UK: Psychology Press; 2001. p. 159-82.

17. Bisiach E, Luzzatti C. Unilateral neglect of representational space. Cortex 1978; 14: 129-33.

18. Chelonis JJ, Edwards MC, Shultz EG, Baldwin R, Blake DJ, Wenger $\mathrm{A}$, et al, Stimulant medication improves recognition memory in children diagnosed with attention deficit/hyperactivity disorder. Exp Clin Psychopharmacol 2002; 10: 400-7.

19. Rhodes SM, Coghill DR, Matthews K. Methylphenidate restores visual memory, but not working memory function in attention deficit disorder. Psychopharmacology 2004; 175: 319-30. 
20. Kitazawa S, Hirabayashi S, Kobayashi M. Memory functions in children with attention deficit/hyperactivity disorder. The effects of methylphenidate in them. No To Hattatsu 2004; 36: 31-6.

21. Scoville WB, Milner B. Loss of recent memory after bilateral hippocampal lesions. J Neurol Neurosurg Psychiatr 1957; 20: 11-21.

22. Rose FC, Symonds CP. Persistent memory defects following encephalitis. Brain 1960; 83: 195-212.

23. Hierons R, Janota I, Corsellis JAN. The late effects of necrotizing encephalitis of the temporal lobes and limbic areas: a clinico-pathological study of 10 cases. Psychological Med 1978; 8: 21-42.

24. Narbona J, Álvarez-Gómez MJ. Limbic-mamillo-thalamic lesion syndromes in childhood and puberty. ANAE Approche Neuropsychologique des Apprentissages chez l'Enfant 1990; 2: 83-7.

25. Vargha-Khadem F, Gadian DG, Watkins KE, Connelly A, Van Paesschen W, Mishkin M. Differential effects of early hippocampal pathology on episodic and semantic memory. Science 1997; 277: 376-80.

26. Gadian DG, Aicardi J, Watkins KE, Porter DA, Mishkin M, Vargha-

\section{TRASTORNOS DE MEMORIA Y DE ATENCIÓN EN DISFUNCIONES CEREBRALES DEL NIÑO}

Resumen. Introducción. La continuidad de las conductas conscientes requiere la puesta en juego de la atención y de la memoria operativa, o memoria de trabajo, para conjugar los datos de la realidad actual con los de la memoria a largo plazo y con los planes secuenciales de acción adaptativa. Pacientes y métodos. Mostramos los resultados preliminares de nuestro estudio de $50 \mathrm{pa}$ cientes con edades entre 7 y 16 años, distribuidos en tres grupos según el trastorno neurocognitivo dominante: grupo TDAH 'puro', grupo TDAH/TA (con trastorno de aprendizaje comórbido) y grupo RMEM (riesgo de trastorno de memoria por daño temporomesial bilateral). A todos se les ha aplicado una batería de pruebas de control atencional y de memoria a corto y largo plazo. Resultados. El CI verbal es superior a 80 en todos los participantes, sin diferencias entre los tres grupos. El grupo TDAH/TA tiene un CI manipulativo y una atención sostenida más bajos que el grupo TDAH, pero no se han objetivado déficit de memoria a largo plazo en ninguno de estos dos grupos. En cambio, el grupo RMEM rinde más bajo en tareas de memoria verbal, a pesar de que su conducta es más atenta y estable, comparativamente con los otros dos grupos. Conclusiones. El análisis neuropsicológico de estas disociaciones permite ajustar las estrategias de intervención terapéutica. El rendimiento en aprendizajes a largo plazo en niños con TDAH y TDAH/TA puede ser mejorado con psicoestimulantes por su efecto activador de la memoria de trabajo. [REV NEUROL 2005; 40 (Supl 1): S33-6]

Palabras clave. Atención. Daño bitemporal. Memoria. Niñez. TDAH.
Khadem F. Developmental amnesia associated with early hypoxicischaemic injury. Brain 2000; 123: 499-507.

27. Maguire EA, Vargha-Khadem F, Mishkin M. The effects of bilateral hippocampal damage on fMRI regional activations and interactions during memory retrieval. Brain 2001; 124: 1156-70.

28. Isaacs EB, Lucas A, Chong WK, Wood SJ, Johnson CL, Marshall C, et al. Hippocampal volume and everyday memory in children of very low birth weight. Pediatr Res 2000; 47: 713-20.

29. Sánchez-Carpintero R, Neville BGR. Attentional ability in children with epilepsy. Epilepsia 2003; 44: 1340-9.

30. Chugani HT, Silva ED, Chugani DC. Infantile spasms: III. Prognostic implications of bitemporal hypometabolism on positron emission tomography. Ann Neurol 1996; 39: 643-9.

31. De Long GR, Heintz ER. The clinical syndrome of early-life bilateral hippocampal sclerosis. Ann Neurol 1997; 42: 11-7.

32. Jambaqué I, Dellatolas G, Dulac O, Signoret JL. Validation de la batterie d'efficience mnésique 144 chez l'enfant d'âge scolaire. ANAE Approche Neuropsychologique des Apprentissages chez l'Enfant 1991; 3: 125-35.

\section{PERTURBAÇÕES DE MEMÓRIA E DE ATENÇÃO EM CRIANÇAS COM DISFUNÇÕES CEREBRAIS}

Resumo. Introdução. A continuidade dos comportamentos conscientes requerem que seja posta em causa a atenção e a memória operativa, ou memória de trabalho, para conjugar os dados da realidade actual com os da memória a longo prazo e com os planos sequenciais da acção adaptativa. Doentes e métodos. Apresentamos os resultados preliminares do nosso estudo envolvendo 50 doentes com idades compreendidas entre os 7 e os 16 anos, distribuídos em três grupos segundo a perturbação neurocognitiva dominante: grupo PDAH 'puro', grupo PDAH/PA (com perturbação de aprendizagem co-mórbida) e grupo RMEM (risco de perturbação de memória devido a lesão temporomesial bilateral). Aplicou-se a todos um conjunto de provas de controlo de atenção e de memória a curto e a longo prazo. Resultados. O CI verbal é superior a $80 \mathrm{em}$ todos os participantes, sem diferenças entre os três grupos. O grupo PDAH/ $P A$ tem um CI manipulativo e uma atenção mantida inferiores ao grupo PDAH, mas não se objectivou o défice de memória a longo prazo em nenhum destes dois grupos. Por outro lado, o grupo RMEM tem um rendimento mais baixo em tarefas de memória verbal, apesar do seu comportamento ser mais atento e estável, comparativamente aos outros dois grupos. Conclusões. A análise neuropsicológica destas dissociações permite ajustar as estratégias de intervenção terapêutica. $O$ rendimento em aprendizagens a longo prazo em crianças com PDAH e PDAH/PA pode ser melhorado com psico-estimulantes pelo seu efeito activador sobre a memória de trabalho. [REV NEUROL 2005; 40 (Supl 1): S33-6]

Palavras chave. Atenção. Infância. Lesão bitemporal. Memória. PDAH. 